\title{
Review of: "Six Month Safety and Efficacy of the BNT162b2 mRNA COVID-19 Vaccine"
}

\author{
Sharon Einav ${ }^{1}$ \\ 1 Shaare Zedek Medical Center
}

Potential competing interests: The author(s) declared that no potential competing interests exist.

This papers shows some interesting findings:

Side effects seem to be similar among young and old. Efficacy also seems similar and two doses do not seem to be harmful. I like the 2 month $6 \%$. That is practical. However, we now need to look forward and the main study limitation is what can (or cannot) be learned from so few severely ill patients:

1. The overall efficacy for prevention of severe COVID is $97 \%$. However, the $\mathrm{Cl}$ for this was relatively broad (80.3-99.9) which may pose a challenge for policy makers. Discuss how many cases need to be randomised to raise the bottom limit of the $\mathrm{Cl}$ to $90-92$.

2. The number of deaths in the non-vaccine group was slightly lower (two patients less). This number will be used by anti-vaxxers. What is the fragility index of the mortality findings?

3. What we need now is a "table 3" specifically for critically ill patients in the two groups (e.g. stratified by age categories and other characteristics). Policy makers need to decide who (and if) should receive additional vaccine doses. Therefore the pertinent question is who became (or will become) severely ill.

4. As this is 6 month data - it is high time to add some information of long term effects (hospital admissions, visits to doctors and clinics, complications) in the two study arms.

5. How do the authors explain no reports of myocarditis?

6. The paper is mostly (perhaps entirely?) written and funded by the company. "Subject to certain criteria, conditions, and exceptions, Pfizer may also provide access to the related individual anonymized participant data" . The data should be unconditionally open to public scrutiny. 